\title{
ABO blood grouping in Egyptian children with rotavirus gastroenteritis
}

\author{
Hala Gouda Elnady ${ }^{1}$, Ola M. Abdel Samie ${ }^{1}$, Maysa Tawhid Saleh ${ }^{1}$, Lobna S. Sherif ${ }^{1}$, Naglaa Abdalmoneam², \\ Naglaa M. Kholoussi ${ }^{3}$, Shams M. Kholoussi ${ }^{2}$, Ahmed N. EL-Taweel ${ }^{4}$ \\ ${ }^{1}$ Medical Division, Child Health Department, National Research Centre, Cairo, Egypt \\ ${ }^{2}$ Paediatrics Department, Faculty of Medicine for Girls, Al-Zahraa University Hospital, Cairo, Egypt \\ ${ }^{3}$ Human Genetics and Genome Research Division, Immunogenetics Department, National Research Centre, Cairo, Egypt \\ ${ }^{4}$ Department of Water Pollution Research, Laboratory of Virology, National Research Centre, Cairo, Egypt
}

Gastroenterology Rev 2017; 12 (3): 175-180

DOI: https://doi.org/10.5114/pg.2017.70469

Key words: gastroenteritis, rotavirus, infants, $A B O$, blood groups.

Address for correspondence: Prof. Maysa Tawhid Saleh, Medical Division, Child Health Department, National Research Centre, El Bouhous St, 00202 Cairo, Egypt, phone: 00201019582252 , e-mail: maytawsal@yahoo.com

\begin{abstract}
Introduction: Rotavirus gastroenteritis is an important public health problem all over the world, causing a notable economic burden in both developing and developed countries.

Aim: To explore the relationship between blood group typing, rotavirus gastroenteritis, and its severity in Egyptian children.

Material and methods: A cross sectional case control study was conducted on 231 cases of acute gastroenteritis attending the outpatient clinic of Al-Zahraa University Hospital. Full history taking, clinical examination, and clinical data collection were done. Blood samples were collected for an ABO grouping. Stool samples were tested for viral gastroenteritis agents.

Results: Rota positive cases of GE were significantly more prevalent among cases with blood group A $(p<0.05)$ and significantly less among cases with blood group B $(p<0.05)$. The rate of hospitalisation was highly significantly greater among cases with group $A(p<0.005)$, and significantly lower among cases with group $A B$ and $O(p<0.05)$. As regards the degree of dehydration, moderate and severe cases were highly significant in groups $\mathrm{A}$ and $\mathrm{O}(p<0.005)$. Rota-positive gastroenteritis showed significant positive correlations with indicators of severity such as hospitalisation, degree of dehydration, and duration of fever $(p<0.005)$.

Conclusions: Blood group A is highly associated with paediatric rotavirus gastroenteritis. This could highlight an important risk factor, which could play a significant role for the pathogenesis of rotavirus gastroenteritis and severity as well. Furthermore, more intervention care could be needed for blood group A paediatric patients, if gastroenteritis especially rotavirus affect this group to avoid comorbidities.
\end{abstract}

\section{Introduction}

Rotavirus (RV) gastroenteritis (GE) is an important public health problem all over the world. It causes high morbidities and considerable hospitalisation rates in infants and young children, with a notable economic burden in both developing and developed countries [1].

$A B O$ blood group system antigens $(A, B$, and $H)$ are complex carbohydrate molecules expressed on the surface of red blood cell membranes [2]. These antigens are also highly expressed on the surface of a variety of human cells and tissues, including the epithelium, sensory neurons, platelets, and the vascular endothelium [3].

The clinical significance of the ABO blood group system extends beyond transfusion medicine. Many studies reported an association between the $\mathrm{ABO}$ blood group system and various human cardiovascular, oncological, and other diseases [4], and with some infectious and non-infectious diseases of the gastrointestinal tract and even other organs $[5,6]$.

Blood group $\mathrm{O}$ was reported to be associated with the occurrence of various diarrheal agents, and infants with blood group $\mathrm{O}$ are more susceptible to diarrheal attacks than those with other blood groups $[6,7]$.

\section{Aim}

The aim of the study was to explore the relationship between blood group typing and Rotavirus gastroenteritis and its severity in Egyptian children. 


\section{Material and methods}

This cross-sectional case control study was conducted on 231 cases of acute gastroenteritis (134 males and 97 females) below 5 years of age, attending the outpatient clinic of Al-Zahraa University Hospital.

Children on corticosteroids or immunosuppressive drug therapy or complaining of systemic autoimmune disease, immunodeficiency, or chronic disease were excluded from the study. Previous history of Rotavirus vaccination as well as blood or any blood product transfusion were also excluded.

The study protocol was approved by the Medical Ethical Committee of the National Research Centre. All parents gained comprehensive and clear knowledge about the aim of our work, and parental approval and written consent was signed by their parents prior to enrolment.

All cases were subjected to full history taking, thorough clinical examination, and collection of clinical data. Clinical data involved disease manifestations such as fever, vomiting, abdominal pain, or bloody diarrhoea. Severity criteria like duration of the diarrhoea, number of stool motions or bouts of vomiting, range of body temperature, and degree of dehydration were determined for all hospitalised children [8].

Blood samples were taken from all cases for an $A B O$ grouping. Blood typing method was used to determine the specific type according to the ABO blood typing system. Blood samples were mixed with antibodies against type $A$ and $B$ blood, and the samples were checked to see whether or not the blood cells agglutinate. This means that the blood reacted with one of the polyclonal antibodies that were obtained from Bio-Rad Laboratories Inc. [9].

Stool samples were collected in dry sterile clean plastic cups. Collected samples were diluted and prepared for RT-PCR for detection of RNA viral gastroenteritis agents in the collected samples. Viral genomes were extracted from $10 \%$ diluted stool samples and prepared for detection of viral gastroenteritis by subjection to extraction of both viral RNA and DNA in the samples using an Axygen ${ }^{\oplus}$ Kit (Axygen biosciences, Cat. No.

Table I. Distribution of ABO grouping in gastroenteritis cases

\begin{tabular}{ccc} 
Blood group & No. of cases & Percent \\
\hline$A$ & 100 & 43.3 \\
\hline$B$ & 37 & 16.0 \\
\hline$A B$ & 32 & 13.9 \\
\hline O & 62 & 26.8 \\
\hline Total & 231 & 100.0
\end{tabular}

AP-MN-BF-VNA-250) to facilitate the detection of the RNA Rotavirus [10].

Cases were divided into two groups according to the type of the viral infection: group 1 Rotavirus-positive gastroenteritis (RV +ve) in 118 cases and group 2 Rotavirus-negative gastroenteritis ( $\mathrm{RV}-\mathrm{ve}$ ) in 113 cases.

\section{Statistical analysis}

Statistical analysis was performed using SPSS version 21. Mean \pm SD was used for age distribution calculations. Chi-square $\left(\chi^{2}\right)$ test was used to compare two non-parametric values. Correlation was done using Pearson correlation. $P$-value $<0.05$ was considered as significant and $p<0.005$ value as highly significant.

\section{Resuls}

The present study was conducted on 231 gastroenteritis children below 5 years of age $(3.4 \pm 1.7$ years), with male to female ratio $1.4: 1$. Out of them, 153 cases were hospitalised.

The distribution of gastroenteritis cases according to $A B O$ grouping is shown in Table I. Blood group A was found to be more prevalent among GE cases (43.3\%) followed by blood group $\mathrm{O}(26.8 \%)$.

Gastroenteritis cases were 118 RV +ve (65 males and 53 females) and 113 RV -ve (69 males and 44 females). There were no significant differences regarding the number or gender distribution in both studied groups $(p<0.05)$ (Table II).

Rota-positive cases were significantly more prevalent among cases with blood group A $(p<0.05)$ and significantly less among cases with blood group $B$ $(p<0.05)$. Both blood groups AB and O showed no statistically significant difference of Rota gastroenteritis $(p>0.05)$ (Table II).

Regarding the degree of dehydration, mild degree was found highly significant in Rota-negative cases of GE $(p<0.005)$ and severe degree was found highly significant in Rota-positive cases of GE ( $p<0.005)$. Moderate degree of dehydration showed no significant difference between Rota positive and negative cases of $\mathrm{GE}(p>0.05)$.

As regards the rate of hospitalisation, it was found to be highly significant more among Rota-positive cases of GE compared to Rota-negative ones ( $p<0.005)$.

Table III showed the distribution of GE cases by ABO grouping according to hospitalisation and degree of dehydration. The rate of hospitalisation was found highly significant more among cases with group A $(p<0.005)$, and significantly less among cases with group $A B$ and $\mathrm{O}(p<0.05)$.

Moreover, moderate and severe dehydration cases were found highly significant among group $\mathrm{A}$ and $\mathrm{O}$ $(p<0.005)$ in both. 
Table II. Distribution of GE cases according to Rotavirus infection

\begin{tabular}{|c|c|c|c|c|}
\hline Variable & Rota positive & Rota negative & $t$ & $P$-value ${ }^{\#}$ \\
\hline Age, mean \pm SD [years] & $3.2 \pm 1.6$ & $3.6 \pm 1.9$ & 0.643 & 0.762 \\
\hline Variable & $N(\%)$ & $N(\%)$ & $\chi^{2}$ & $P$-value* \\
\hline$N$ of GE cases & $118(51.1)$ & $113(48.9)$ & 0.108 & 0.742 \\
\hline \multicolumn{5}{|l|}{ Gender: } \\
\hline Males & $65(48.5)$ & $69(51.5)$ & 0.119 & 0.730 \\
\hline Females & $53(54.6)$ & $44(45.4)$ & 0.835 & 0.361 \\
\hline \multicolumn{5}{|l|}{ Blood grouping: } \\
\hline A blood group & $61(61.0)$ & $39(39.0)$ & 4.840 & 0.028 \\
\hline B blood group & $12(32.4)$ & $25(67.6)$ & 4.568 & 0.033 \\
\hline AB blood group & $16(50.0)$ & $16(50.0)$ & 0.118 & 0.732 \\
\hline O blood group & $29(46.8)$ & $33(53.2)$ & 0.670 & 0.796 \\
\hline \multicolumn{5}{|l|}{ Degree of dehydration: } \\
\hline Mild & $17(14.4)$ & $43(38.1)$ & 10.246 & 0.001 \\
\hline Moderate & $52(44.1)$ & $48(42.5)$ & 0.160 & 0.689 \\
\hline Severe & $49(41.5)$ & $22(19.5)$ & 9.657 & 0.002 \\
\hline Hospitalisation & 106/118 (89.8) & 47/113 (41.6) & 4.658 & 0.004 \\
\hline
\end{tabular}

"By Student $t$ test ( $(t),{ }^{*}$ by $\chi^{2}$ test.

Table III. Distribution of GE cases by ABO grouping according to hospitalisation and degree of dehydration

\begin{tabular}{lcccc} 
Hospitalisation & $\begin{array}{c}\text { Not hospitalised } \\
(\boldsymbol{N}=\mathbf{7 8})\end{array}$ & $\begin{array}{c}\text { Hospitalised } \\
(\boldsymbol{N}=\mathbf{1 5 3})\end{array}$ & $\chi^{\mathbf{2}}$ & $\boldsymbol{P}$-value \\
\hline A blood group & $29(29.0 \%)$ & $71(71.0 \%)$ & 8.640 & 0.002 \\
\hline B blood group & $15(40.5 \%)$ & $22(59.5 \%)$ & 1.324 & 0.250 \\
\hline AB blood group & $11(34.4 \%)$ & $21(65.6 \%)$ & 9.529 & 0.020 \\
\hline O blood group & $23(37.1 \%)$ & $39(62.9 \%)$ & 5.400 & 0.020 \\
\hline Dehydration & $\begin{array}{c}\text { Mild } \\
(\boldsymbol{N}=\mathbf{6 0})\end{array}$ & $\begin{array}{c}\text { Moderate/severe } \\
(\boldsymbol{N}=171)\end{array}$ & $\chi^{2}$ & $\boldsymbol{P}$-value \\
\hline A blood group & $27(27.0 \%)$ & $73(73.0 \%)$ & 18.360 & $<0.001$ \\
\hline B blood group & $11(29.7 \%)$ & $26(70.3 \%)$ & 6.081 & 0.014 \\
\hline AB blood group & $8(25.0 \%)$ & $24(75.0 \%)$ & 7.529 & 0.006 \\
\hline O blood group & $14(22.6 \%)$ & $48(77.4 \%)$ & 19.267 & $<0.001$
\end{tabular}

By $\chi^{2}$ test.

Table IV. Correlations between Rota virus infection and ABO grouping, hospitalisation, degree of dehydration, and fever in GE cases

\begin{tabular}{lcccc} 
Rota GE & ABO & Hospitalisation & Dehydration & Fever \\
\hline Pearson correlation & 0.101 & $0.516^{\star *}$ & $0.304^{* *}$ & $0.456^{\star *}$ \\
\hline Sig. (2-tailed) & 0.126 & 0.000 & 0.000 & 0.000
\end{tabular}

${ }^{* *}$ Correlation is significant at the 0.001 level (2-tailed).

Rota-positive gastroenteritis showed significant positive correlations with the indicators of severity such as hospitalisation, degree of dehydration, and duration of fever ( $p$ < 0.001). However, Rota-positive gastroenteritis showed statistically non-significant correlation with the ABO blood grouping ( $p=0.126$ ) as shown in Table IV. 


\section{Discussion}

Rotavirus is considered the most common cause of severe gastroenteritis in infants and young children worldwide and of diarrheal mortality in developing countries [11]. The World Health Organisation (WHO) reported an annual mortality rate of more than half a million children under the age of 5 years from rotavirus disease [12]. In Egypt, an annual prevalence of Rotavirus gastroenteritis of $42 \%$ among the reported episodes of gastroenteritis below five years of age was reported in a study covering the WHO Eastern Mediterranean region [13]. Rotavirus was reported as one of the most common viruses circulating in the environment and in the population in great Cairo $[14,15]$.

Blood group frequencies were shown to differ between populations. The distribution of the main $A B O$ blood groups in the Egyptian population was reported as $36.44 \%$ for blood group 0, 33.94\% for group $A$, $20.96 \%$ for blood group $B$, and $8.65 \%$ for group $A B[16]$.

The present study showed that blood group $A$ was encountered more among gastroenteritis cases (43.3\%) followed by blood group $\mathrm{O}(26.8 \%)$, although blood group $\mathrm{O}$ is the predominant group among the Egyptian population. Moreover, $61 \%$ of the GE cases with blood group A were found to be Rota-positive cases; $71 \%$ of them were hospitalised and $73 \%$ were suffering from moderate to severe forms of dehydration. Those results suggest that blood group A may be a receptor and host-susceptibility factor for the rotavirus gastroenteritis in the Egyptian paediatric population, as observed in previous studies $[17,18]$.

Host susceptibility to specific human Rotavirus strains and the pathogenesis are influenced by the genetically controlled expression of the different human blood group antigens (HBGAs). The "A type" HBGAs are genetically determined glycoconjugates present in RBCs, mucosal secretions, and epithelial cells. HBGAs are recognised as susceptibility and cell attachment factors for gastric pathogens like Noro viruses and Helicobacter pylori $[17,19]$. Similarly, a very recent study in Spain reported that the A type HBGAs are receptors for human RVs (although RV different strains vary in their ability to recognise these antigens) [20].

Again, another recent study by Tan and Jiang also reported that Rotavirus and Norovirus recognise HBGAs as receptors or ligands for attachment. The human HBGAs are highly polymorphic and contain $A B O$, secretor, and Lewis antigens [21].

Moreover, some researchers found that cells engineered to express the " $A$ " antigens on their surface were easily infected by the RV strains. They also reported that blocking the A antigen prevented Rotavirus infection in human intestinal cells $[17,19]$.
Consistent with our results, some studies declared that the A-type blood group was more common among the RV +ve GE group than in the RV -ve group [22]. The hospitalisation rates of blood group $A$ patients were even higher. Some researchers also suggested that the "A" type blood group may be a receptor and host susceptibility factor for RV-GE $[17,18]$.

However, Hamza et al. are not in agreement with our results, where no correlation was found between the risk of RV-GE and type "A" blood group or even any other type of blood group $(p>0.05)$ [23].

In our study, 62 (26.8\%) out of 231 cases of GE belonged to the $\mathrm{O}$ blood group. This is not a small number and it comes in the second rank after the prevailing group A (43\%).

Similarly, some studies reported that patients with O-type blood group were more susceptible to GIT infection (but by E. coli) [24, 25].

Several possible explanations to why a respectful percentage of the GE cases were of the O-type blood group were reported by several studies [26]. This could be due to the loss of the functional proteins "A/B transferase" in type O blood group patients, which would have some deleterious consequences on these patients. Moreover, possession of a homozygosity for a "non-secretor phenotype" has a demonstrable survival advantage for some infectious diseases [24]. In Norovirus infections, some of the different strains of noroviruses were reported to bind to "non-secretors Le $\mathrm{e}^{\mathrm{a}-\mathrm{b} \text { " }}$ structures and cause symptomatic infection [27]. Thus the non-secretors phenotype could give some explanation for the presence of a remarkable number of cases of GE to be O blood group type.

Besides the presence of a gene coding for the blood type, there also exists a separate gene determining every person's ability to secrete his blood type antigens into the body fluids and secretions [26]. Being a secretor or a non-secretor is completely independent of the type of his blood group. Research studies have reported that not only is there a strong relationship between individuals' susceptibility to some diseases and their ABO blood groups but also their secretor status [26]. Furthermore, there are reports that the ability to secrete blood type antigens into body fluids and secretions gives an "additional degree of protection" against microorganisms, lectins, and the environment generally [28]. Again, they noted that since secretors have a steady supply of their blood type in their GIT mucus lining, their intestinal friendly probiotic bacteria ecosystem is almost always in a stabilised condition [28].

This issue of secretors and non-secretors might be of great help in explaining why in our control group (RV -ve GE cases) the results were unexpected. The most 
commonly found blood group among them was the " $A$ " blood group (39) followed by "O" (33) then "B" (25) and finally $A B(16)$. Here we would like to draw attention to two important observations.

First: This distribution is not exactly like the $A B O$ blood group distribution in the general population, but we can say that it is more or less approaching it. The reason for this might be that the sample taken was small (number of cases is 231).

Second: What was really surprising was that although we had the "A" and "O" blood groups involving the highest incidence of RV +ve GE cases, still the largest numbers of the RV -ve GE cases were also confined between those two groups. This can be explained through resorting to the "Secretors and non-secretors" issue. Accordingly, we can say that the majority of the RV +ve GE cases belonged to the "A" type blood group but were "non-secretors". On the other hand, the majority of the RV -ve GE cases who were "A" type were most probably secretors. The same explanation applies to why considerable numbers of RV +ve GE cases and RV -ve GE ones both belonged to the O-type blood groups. Moreover, we can say that blood group $\mathrm{A}$ followed by $\mathrm{O}$ are risk groups for gastroenteritis especially RV.

However, Throven et al. confirmed that Norovirus GE patients who are homozygous for "non-secretors" were protected from infection, and this clearly demonstrates the pivotal role of "secretor status" in determining the susceptibility to Noroviruses (and maybe similarly we can apply this to RV-GE) [29].

Again, Larrison et al. clearly showed lower antibody titres to Noro VGGII in non-secretors compared to secretors [30].

On the other hand, some researchers reported that there are obvious examples of protection against Noroviruses because of inheritance of polymorphisms in the genes encoding and regulating the expression of the $\mathrm{ABH}$ and lewis antigens in body secretions [24].

Although we did not investigate the secretor and non-secretor status of our GE cases and of our control group children, until further research is done the former research study reports could be a very possible proper explanation for our results.

Finally, we would like to explain that in our study we resorted to explanations concerning Noroviruses because of the following reasons:

Like RVs, Noroviruses are a principal cause of GE in developing countries and a very recent study has proved that histo blood group A phenotype is a key susceptibility factor for both Norovirus and RV infections in children [18].

Many studies have reported that co-infections of RVs and Noroviruses were the most commonly met [24, 27].
The RVs have different strains like Noroviruses, and both viruses are transmitted by water and food contamination [14, 31].

\section{Conclusions}

Blood group A was highly associated with paediatric rotavirus gastroenteritis in the Egyptian community. This could highlight an important risk factor, which could play a significant role for the pathogenesis of rotavirus gastroenteritis and severity as well. Furthermore, more interventional care could be needed for (blood group A) paediatric patients, if gastroenteritis especially rotavirus affect this group to avoid comorbidities. Moreover, we need more research studies concerning the influence of blood group on susceptibility to gastroenteritis. This might be of great help in evading problems.

\section{Conflict of interest}

The authors declare no conflict of interest.

\section{References}

1. Bekdas M, Demircioglue F, Goksugur SB, et al. Is there a relationship between rotavirus positive gastroenteritis and $A B O$ blood groups: a retrospective cohort study? A retrospective cohort study. Arch Argent Pediatr 2014; 112: 345-8.

2. Storry JR, Olsson ML. The ABO blood group system revisited: a review and update. Immunohematology 2009; 25: 48-59.

3. Franchini M, Capra F, Targher G, et al. Relationship between ABO blood group and von Willebrand factor levels: from biology to clinical implications. Thromb J 2007; 5: 14.

4. Franchini M, Favaloro EJ, Targher G, et al. ABO blood group, hypercoagulability, and cardiovascular and cancer risk. Crit Rev Clin Lab Sci 2012; 49: 137-49.

5. Vogel F. Controversy in human genetics: ABO blood groups and disease. A J Human Genetics 1970; 22: 464-75.

6. Masoumi JP, Mozoni S. The relationship between ABO blood groups and susceptibility to diarrhea in infants during the first year of life. MJIRI 2003; 16: 239-43.

7. Boren T, Falk P, Roth KA, et al. Attachment of Helicobacter pylori to human gastric epithelium mediated by blood group antigens. Science 1993; 262: 1892-5.

8. World Gastroenterology Organization Global Guidelines. Acute diarrhea in adults and children: a global perspective in February 2012.

9. Davenport RD. Transfusion medicine. In: Henry's Clinical Diagnosis and Management by Laboratory Methods. McPherson RA, Pincus MR (eds.). $22^{\text {nd }}$ edn., Saunders 2011.

10. El-Nady HG, Sherif LS, Saleh MT, et al. Basic science prediction of gut wall integrity loss in viral gastroenteritis by non-invasive marker. Maced J Med Sci 2015; 3: 37-45.

11. Widdowson MA, Steele D, Vojdani J, et al. Global rotavirus surveillance: preparing for the introduction of rotavirus vaccines. J Infect Dis 2009; 200 (Suppl 1): S1-8.

12. Parashar UD, Burton A, Lanata C, et al. Global mortality associated with rotavirus disease among children in 2004. J Infect Dis 2009; 200 (Suppl 1): S9-15. 
13. Teleb N. Rotavirus surveillance network in the Eastern Mediterranean region. Presented at the $8^{\text {th }}$ International Rotavirus Symposium. 2008 June 3-4 Istanbul.

14. Kamel AH, Ali MA, El-Nady HG, et al. Predominance and circulation of enteric viruses in the region of Greater Cairo, Egypt. J Clin Microbiol 2009; 47: 1037-45.

15. Kamel AH, Ali MA, El-Nady HG, et al. Evidence of the co-circulation of enteric viruses in sewage and in the population of greater Cairo. J Appl Microbiol 2010; 108: 1620-9.

16. Shaik YA, Alhawary AS, Shbair AS, et al. Frequency of ABO and $\mathrm{Rh}$ (D) blood groups in five governorates and Gaza strip. Pak J Med Sci 2007; 23: 924-7.

17. Hu L, Crawford SE, Czako R, et al. Cell attachment protein VP8* of a human rotavirus specifically interacts with A-type histo-blood group antigen. Nature 2012; 485: 256-9.

18. Van Trang N, Vu HT, Huang P, et al. Association between Norovirus and Rotavirus infection and histo-blood group antigen types Vietnamese children. J Clin Microbiol 2014; 52: 1366-74.

19. Glass RI, Parashar UD, Estes MK. Norovirus gastroenteritis. N Engl J Med 2009; 361: 1776-85.

20. Bohm R, Fleming FE, Maggioni A, et al. Revisiting the role of histo-blood group antigens in rotavirus host-cell invasion. Nat Commun 2015; 6: 5907.

21. Tan M, Jiang X. Histo-blood group antigens: a common riche for Norovirus and Rota virus. Expert Rev Mol Med 2014; 16: e5.

22. Bekdas M, Demircioglu F, Goksugur FB, et al. Is there a relationship between rotavirus positive gastroenteritis and $A B O$ blood groups? A retrospective cohort study. Arch Argent Pediatr 2014; 112: 345-8.

23. Hamza Y, Esengul K, Arzu G, et al. Blood group and Rotavirus gastroenteritis. Pediatr Infect Dis J 2013; 32: 705-6.

24. Anstee DJ. The relationship between blood groups and disease. Blood J 2010; 115: 4635-43.

25. Blackwell CC, Dundas S, James VS, et al. Blood group and susceptibility to disease caused by Escherichia coli 0127. J Infect Dis 2002; 185: 393-6.

26. Jaff MS. Higher frequency of secretor phenotype 0 blood group, its benefits in prevention and or treatment of some diseases. Int J Nanomed 2010; 5: 901-5.

27. Carlsson B, Kindbrg E, Buesa J, et al. The G428A non sense mutation in FUT2 provides strong but not absolute protection against symptomatic G11.4 Norovirus infection. Plos One 2009; 4: e5593.

28. Dr. Peter D’adamo's Book. Eat Right 4 your blood type. 2009 www.dadamo.com.

29. Throven M, Grahn A, Hedlund K, et al. A homozygous nonsense mutation (428G-A) in the human secretor (FUT 2) gen provides resistance to symptomatic norovirus (CG II) infections. J Virol 2005; 79: 15351-5.

30. Larisson MM, Rydell GE, Grahn A, et al. Antibody prevalence and titre to norovirus (genogroup II) correlate with secretor (FUT 2) but not with ABO phenotype or Lewis (FUT 3) genotype. J Infect Dis 2006; 194: 1422-7.

31. Zaghloul MZ, El-Sahn SF, Galal ZA. Confection of Rotavirus group A, Norovirus and Adenovirus in Egyptian children with gastroenteritis. Life Sci J 2013; 10: 848-52.

Received: 9.11.2015

Accepted: 2.03 .2016 